\title{
Telemedicina y pediatría en la pandemia de COVID-19
}

\author{
Telemedicine and pediatrics in the COVID-19 pandemic \\ José Luis Pinacho Velázquez, * Mariana Pinacho Juárez, ${ }^{\ddagger}$ Manuel Ángel Correa Flores ${ }^{\S}$ \\ Citar como: Pinacho VJL, Pinacho JM, Correa FMÁ. Telemedicina y pediatría en la pandemia de COVID-19. \\ Acta Med Grupo Angeles. 2021; 19 (2): 258-261. https://dx.doi.org/10.35366/100451
}

\section{Resumen}

Durante el brote de COVID-19, el confinamiento ha significado un desafío que enfrentan los profesionales de la salud, y los pediatras no son la excepción. Sean telefónicas o de video, las consultas remotas desempeñan un papel clave en esta pandemia, tales herramientas dan información válida para el diagnóstico y tratamiento de enfermedades. En este artículo se describe cómo la telemedicina es una opción en la atención médica, tanto en pacientes con COVID-19, como con otros trastornos. Se describe la manera en la que los pediatras pueden hacer uso de esta herramienta y se relata la experiencia a nivel internacional. Los pediatras deben estar familiarizados con el uso de esta tecnología, a fin de poder ofrecer cuidado médico por videollamada.

Palabras clave: Telemedicina, videollamadas, pediatría, COVID-19.

El nuevo brote de coronavirus SARS-CoV-2 es altamente contagioso, por lo que se han implementado nuevos modelos de atención médica, buscando evitar la entrevista presencial, y reduciendo así el riesgo de propagación del virus. Los gobiernos a nivel internacional, incluido el de México, por recomendación de la Organización Mundial de la Salud han introducido medidas de cierre de actividades de la población, que incluyen distanciamiento social

\footnotetext{
* Médico pediatra urgenciólogo.

* Médico general. Universidad Anáhuac.

$\S$ Pediatra intensivista.
}

Hospital Ángeles Lindavista.

Correspondencia:

José Luis Pinacho-Velázquez

Correo electrónico: joselpinacho@hotmail.com

Aceptado: 05-05-2021.

www.medigraphic.com/actamedica

\section{Abstract}

Confinement during the COVID-19 outbreak has posed a challenge for healthcare professionals, and pediatricians are no exception. Telephone or video consultations play a key role in this pandemic, these tools provide valid information for the diagnosis and treatment of diseases. This article describes how telemedicine is an option in medical care, both in patients with COVID-19, and with other diseases. The way in which pediatricians can make use of this tool and the international experience are described. Pediatricians must be familiar with the use of this technology, in order to offer medical care by video call.

Keywords: Telemedicine, videocalls, pediatrics, COVID-19.

y confinamiento, mediante las cuales se restringe el movimiento de las personas con afectación de la vida diaria. ${ }^{1,2}$

Las estrategias de telecomunicaciones, como las consultas telefónicas o de video, desempeñan un papel clave en esta pandemia, obteniendo mediante estas herramientas información válida para el diagnóstico y tratamiento de enfermedades. ${ }^{3,4}$

Existe una buena práctica de telemedicina que establece claramente el papel y las limitaciones de las teleconsultas. ${ }^{5}$ La telemedicina (TM) tiene un papel en evolución en diversas especialidades y la pediatría no es la excepción.

El Servicio Nacional de Salud (NHS) del Reino Unido ha implementado programas basados en Internet como la tecnología digital Attend Anywhere apoyada y promovida por ellos en la actual pandemia, mediante entrevistas por video, ${ }^{6,7}$ con lo cual se reconoce que las consultas de telemedicina (TM) o remotas son de vital importancia en el ofrecimiento de asistencia medica ahora y en el futuro.

La Organización Mundial de la Salud (OMS) definió a la TM como la prestación de servicios de asistencia médica, donde la distancia es un factor crítico por parte de 
los profesionales de la medicina, utilizando tecnologías de información y comunicación para el intercambio de detalles válidos para el diagnóstico, tratamiento y prevención de enfermedades o lesiones, investigación y evaluación además de educación continua de los trabajadores sanitarios, con el objetivo de promover la salud de las personas y las comunidades.

Es importante que en la República Mexicana se adopten lineamientos de buenas prácticas médicas, procurando librarse de las malas al realizar reuniones por TM o telefónicas.

La pandemia de COVID-19 ha modificado el uso de las herramientas electrónicas en varios campos del quehacer humano, sobre todo en el educativo y de salud, por lo que es necesario que en México sean adaptadas de manera apropiada a los marcos legales existentes, así como promover el uso de este tipo de utilerías. Mas han de realizarse con responsabilidad, pues no es lo mismo revisar al paciente en forma directa, tampoco debe usarse intentando disminuir costos, y no debería ser un incentivo para incrementar los ingresos del personal de salud.

La telemedicina no es algo nuevo, se ha acelerado a raíz de la pandemia por COVID-19, lo mismo puede ser utilizada como herramienta dentro de las diferentes especialidades de la medicina, incluida la pediatría.

Es responsabilidad del galeno el resguardo de datos personales en posesión de particulares y de protección de datos confidenciales en poder de sujetos obligados. Por lo tanto, es necesario tener el consentimiento informado, en donde se le deben explicar al paciente aspectos tales como la posibilidad de fallas tecnológicas, la forma de prescripción de medicamentos, las interconsultas que se pudieran generar, además de tener un registro detallado de que el enfermo, o la persona responsable del mismo, entendió las recomendaciones de la sesión.

En este país, el Centro Nacional de Excelencia Tecnológica en Salud (CENETEC) es el organismo encargado de emitir las reglamentaciones en este campo y existe una cédula oficial de instrumentos jurídicos para la telesalud, cuyo marco legal tiene su origen en el derecho constitucional y la Ley General de Salud.

La tecnología debería considerarse sólo como una herramienta capaz de fortalecer las necesidades y retos que enfrentan los servicios de salud, tomando en cuenta tres aspectos básicos:

1. Seguridad del paciente.

2. Seguridad del profesional de salud.

3. Seguridad en la transmisión de la información. ${ }^{8}$

La adopción de buenas prácticas médicas y evitar las malas prácticas, al realizar consultas por videollamadas, es parte de la ética de los médicos.
Se recomienda:

1. Seguir los principios de la ética médica.

2. Proteger la privacidad y confidencialidad del paciente.

3. Confirmar que la configuración del equipo remoto o video funcione correctamente.

4. Confirmar que el motivo de la sesión remota es apropiado para el entorno clínico "Apretón de manos virtual y confirmar identidad".

5. Verificación de la anuencia verbal al realizar consultas a distancia.

6. Realizar una evaluación completa de los síntomas y signos virtuales del enfermo. Resumir la entrevista y asegurarse de que el paciente comprenda el plan de manejo.

7. Permitir al paciente la oportunidad de aclarar.

8. Dar seguimientos apropiados.

9. Cuando sea necesario, comunicarse con el facultativo que refiere.

Qué no se debe hacer:

1. No recetar medicamentos de la lista restringida específica, por ejemplo opioides, sin autenticación adecuada.

2. Nunca usar mal las imágenes y los datos del paciente.

3. No recetar medicamentos sin un diagnóstico apropiado o provisional.

\section{PAPEL Y LIMITACIONES DE LAS CONSULTAS CON MEDICAMENTOS}

La TM ha revolucionado la forma en que se brinda auxilio médico en éste y en otros países, especialmente en el contexto de la COVID-19, con la necesidad de prescindir de la consulta "cara a cara" $y$, por lo tanto, reducir el riesgo de transmisión de enfermedades. ${ }^{6,9,10}$ Se ha aplicado en otras especialidades como endocrinología, asistencia oncológica y oftalmología. ${ }^{10,11}$ En dolencias crónicas, como la diabetes y la hipertensión, permite la monitorización remota. ${ }^{12}$ Sin embargo, la implementación de la tecnología TM en países como la India, y México es un caso similar, no ha estado exenta de problemas inherentes, ${ }^{13}$ con varios obstáculos para los usuarios finales que deben ser negociados y que se deben evitar a fin de prevenir problemas médicolegales. ${ }^{14}$ Se pueden realizar avances tendientes a aprovechar las instalaciones de TM, entregando una atención eficaz y segura al paciente. Se destacan los estándares de práctica hacia una telemedicina efectiva y los pasos que se pueden tomar a fin de esquivar las trampas comunes. 


\section{SUGERENCIAS DE PRÁCTICA PARA CONSULTAS EFECTIVAS DE TELEMEDICINA Y PREVENCIÓN DE DIFICULTADES}

Formación. La capacitación y el aprendizaje de habilidades (competencias) en el manejo de consultas remotas es esencial antes de poder proporcionar una atención efectiva y segura al afectado. La capacitación asegurará una comunicación adecuada, un triaje, una mayor eficiencia y la satisfacción del paciente.

Los principios básicos de buenas prácticas médica se aplican a consultas de videollamadas como a cualquier otra, como en las entrevistas personales ${ }^{8,12}$ durante esta pandemia de COVID-19.

Consentimiento. Un asentimiento válido es imprescindible durante una consulta por videollamada y, por lo tanto, se requiere una aprobación explícita del paciente si un trabajador de salud o un cuidador aspira a iniciar una reunión de TM. ${ }^{7,10}$

$\mathrm{Si}$ el paciente comienza la consulta de TM, entonces el consentimiento debería estar implícito. Aunque la conformidad puede estar implícita, es crucial salvaguardar la información personal y confidencial. Se tiene que advertir al enfermo sobre la limitación de la sesión remota. ${ }^{5,10} \mathrm{La}$ capacidad del paciente para tomar decisiones debe ser evaluada y registrada. Por ejemplo, "Sí, consiento en hacer uso de la consulta por telemedicina".

Confidencialidad. Los principios de ética médica, incluidas las normas profesionales que buscan proteger la privacidad y la confidencialidad del afectado según las leyes, serán vinculantes y deberán respetarse y practicarse. La consulta remota se lleva a cabo en un entorno apropiado con consideración de privacidad y datos confidenciales. Se debe asegurar al paciente sobre la naturaleza de la atención remota y que la conversación es segura y confidencial.

Protocolos sugeridos. Preparación y confirmación de los detalles del paciente antes de la consulta "Apretón de manos virtual". Introducción del clínico. Evaluación clínica adecuada. Resumen de los planes de manejo discutidos procurando evitar confusiones y malas interpretaciones. Explicación de los planes de seguimiento. Permitir que el paciente aclare o pregunte.

Documentación (expediente). Se obtiene el consentimiento en caso de que la conversación se registre según las instrucciones..$^{15}$ Estas grabaciones formarán parte de los registros médicos de los pacientes y deben almacenarse de forma segura. El asentimiento para la grabación está documentado en los registros. Una limitación importante frente al uso de TM es tomar decisiones clínicas erróneas (a veces), debido a la falta de examen del paciente y depender de sus informes de investigación e historial. ${ }^{12} \mathrm{~A}$ fin de compensar la falta de examen clínico, es esencial asegurar un expediente para minimizar el riesgo potencial de consultas remotas, que los doctores están ansiosos por esquivar, incluido un plan de manejo acordado y la confirmación de las evaluaciones de seguimiento.

Fotografías clínicas y orientación. Los mismos principios de ética en medicina se aplican a las fotografías e impresiones visuales, como a grabaciones de audio especialmente relacionadas con partes sensibles del cuerpo, niños y, por lo mismo, se toma el consentimiento apropiado y se protege la confidencialidad. ${ }^{15}$

Comunicación. Se ha de enviar una copia de la consulta al paciente, y al médico de referencia del paciente, para la continuidad de la atención. ${ }^{16}$

Red de seguridad. La consulta remota debe permitir la "estratificación del riesgo" y, si es necesario, se organiza una cita presencial para permitir la evaluación clínica, p. ej. una lesión cutánea sospechosa que no se valora en forma adecuada en una asistencia por videollamada, al desear descartar una malignidad o erupción cutánea atípica en un niño para descartar una infección meningocócica grave. ${ }^{17}$

Protección de información y de datos. Los datos se hacen, almacenan, transfieren, protegen o eliminan según las leyes de protección de datos, para evitar posibles infracciones.

Plataforma por utilizar. Es importante la plataforma de tecnología que se va a usar ya que no todas ellas ofrecen la misma funcionalidad. Varias aplicaciones desarrolladas originalmente para videoconferencias (Zoom, Microsoft Teams y Skype for business), o de mensajería como Skype, WhatsApp y FaceTime, podrían utilizarse en las consultas por videollamada. Los programas están disponibles para descargarse gratuitamente o bien lo están a través de licencias organizacionales.

\section{CONCLUSIÓN}

La COVID-19 ha hecho que los médicos, en circunstancias desafiantes, pasen rápidamente de las citas tradicionales "presenciales" a las consultas telefónicas o por video. La capacitación adecuada y el seguimiento de los principios básicos de buenas prácticas médicas basadas en los principios de la bioética, el expediente, la comunicación y la observación del manejo de la información ayudarán en gran 
medida a evitar las dificultades asociadas con las sesiones por videollamada. La revisión periódica de entrevistas por videollamada, incluidas la evaluación y comentarios de los pacientes, reforzará la práctica clínica. La tecnología de la telemedicina es un factor clave en la prestación de atención médica en este presente y en el futuro, y se debe garantizar su práctica correcta durante las consultas por videollamada, así reducirá quejas y problemas médicolegales asociados con ellas.

\section{REFERENCIAS}

1. World Health Organization. Coronavirus disease (COVID-19) advice for the public. [Accessed 22 May 2020] Available in: https://www.who. int/emergencies/diseases/novel-coronavirus-2019/advice-for-public

2. Centers for Disease Control and Prevention. [Accessed 20 Jun 2020] Available in: https://www.cdc.gov/ coronavirus/2019-nCoV/index.html

3. Van Galen LS, Car J. Telephone consultations. BMJ. 2018; 360: k1047.

4. Greenhalgh T, Wherton J, Shaw S, Morrison C. Video consultations for covid-19. BMJ. 2020; 368: m998.

5. Ministry of Health and Family Welfare, Government of India. Telemedicine practice guidelines. Available in: https://www.mohfw. gov.in/pdf/Telemedicine.pdf

6. NHS England and NHS Improvement. Attend anywhere. [Accessed 22 jul 2020] Available in: https://england.nhs. attendanywhere.com/ resourcecentre/Content/Public Topics/Discover.htm

7. Remote consultations. NHS England and NHS improvement coronavirus. Specialty guides for patient management. [Accessed 22 May 2020] Available in: https://www.england. nhs.uk/coronavirus/ publication/specialty.guides

8. Cédula de Instrumentos Jurídicos aplicables a la práctica de la Telesalud en México. [Recurso electrónico]. México: Secretaría de Salud, Centro Nacional de Excelencia Tecnológica en Salud; 2019.

9. General Medical Council. Good medical practice. Updated 29 April 2019, [Accessed 22 May 2020] Available in: https://www.gmc-uk.org/ ethical-guidance/ethical-guidance-for-doctors/good-medical-practice

10. Mayadevi M, Thankappan K, Limbachiya SV, Vidhyadharan S, Villegas B, Ouyoung M et al. Interdisciplinary telemedicine in the management of dysphagia in head and neck. Dysphagia. 2018; 33 (4): 474-480. Available in: https://doi.org/10.1007/s00455-018-9876-9

11. Banerjee M, Chakraborty S, Pal R. Teleconsultation and diabetes care amid COVID-19 pandemic in India: scopes and challenges. J Diabetes Sci Technol. 2020; 14 (4): 714-715. Available in: https:// doi.org/10.1177/1932296820929391

12. Vaishya R, Bahl S, Singh RP. Letter to the editor in response to: telemedicine for diabetes care in India during COVID19 pandemic and national lockdown period: guidelines for physicians. Diabetes Metab Syndr. 2020; 14 (4): 687-688. Available in: https://doi. org/10.1016/j.dsx.2020.05.027

13. Sood SP. Implementing telemedicine technology: lessons from India. World Hosp Health Serv. 2004; 40 (3): 29-43.

14. Chellaiyan VG, Nirupama AY, Taneja N. Telemedicine in India: where do we stand? J Family Med Prim Care. 2019; 8 (6): 1872-1876. Available in: https://www.jfmpc.com/article.asp?issn=2249-4863; ye ar $=2019 ;$ volume $=8 ;$ issue $=6 ;$ spage $=1872 ;$ epage $=1876$; aulast $=C$ hellaiyan

15. General Medical Council (GMC). Guidance for doctors. [Accessed 9 May 2020] Available in: https://www.gmc-uk.org/ethical-guidance/ ethical-guidance-for-doctors/making-and-using-visual-and-audiorecordings-of-patients

16. Stone JH. Communication between physician and patients in the era of e-medicine. N Engl J Med. 2007; 356: 2451-544.

17. Katz HP, Kaltsounis D, Halloran L, Mondor M. Patient safety and telephone medicine: some lessons from closed claim case review. J Gen Intern Med. 2008; 23 (5): 517-522. Available in: https://doi. org/10.1007/s11606-007-0491-y 